\title{
AUTHOR INDEX (Volume 21)
}

\author{
Achour, A. 1450059 \\ Ahsan, B. 1450059 \\ Alam, M. 1450059 \\ Alelaimi, M. 1450034 \\ Ali, M. 1450048 \\ Anandan, C. 1450054 \\ Ansari, M. S. 1450005 \\ Attar, M. M. 1450088 \\ Azizi, S. 1450073 \\ Ba, Z. X. 1450085 \\ Bahari, A. 1450080 \\ Bai, Y. 1450009 \\ Bartholomew, C. 1450060 \\ Bera, P. 1450054 \\ Besant, R. W. 1450044 \\ Bhadane, H. 1450046 \\ Bharuth-Ram, K. 1450012 \\ Bouafia, F. 1450072 \\ Bouziane, K. 1450001 \\ Burghaus, U. 1450060 \\ Cai, F. 1450035 \\ Cai, Y. 1450040 \\ Cao, W. 1450058 \\ Chakraborty, A. 1450049 \\ Chakradhar, A. 1450060 \\ Chari, R. 1450005 \\ Chen, C. Z. 1450026 \\ Chen, J. 1450003 \\ Chen, N. 1450002 \\ Chen, S. $\quad 1450068,1450078$ \\ Chen, X. 1450003 \\ Chen, Y. 1450003 \\ Cheng, H. 1450071 \\ Cheng, L. 1450051, 1450066, \\ 1450068, 1450078 \\ Chikouche, I. 1450082 \\ Chung, G.-S. $\quad$ 1450050, 1450086 \\ Cui, N. Y. 1450055 \\ Cui, S. 1450077 \\ Cui, Y. 1450031 \\ Dai, X. H. 1450029,1450079 \\ Das, N. 1450022 \\ Derbali, L. 1450041 \\ Dimassi, W. 1450041 \\ Ding, K. 1450077 \\ Ding, X. 1450077 \\ Dizaji, H. R. 1450073 \\ Dong, C. 1450023 \\ Dong, L. 1450023 \\ Du, X. 1450027 \\ Duan, R.-S. 1450063 \\ Ehsani, M. H. 1450073 \\ Emetere, M. E. 1450075 \\ Eswaramoorthi, V. 1450020 \\ Evitts, R. W. 1450044 \\ Ezzaouia, H. 1450041 \\ Fan, X. 1450087 \\ Fang, L. 1450033 \\ Fang, X. 1450087 \\ Fang, Z. 1450071 \\ Feng, C. 1450064 \\ Feng, Q. 1450076 \\ Gao, P.-J. 1450053 \\ Gao, Y. 1450071 \\ Gautam, D. K. 1450046 \\ Ge, J. 1450038 \\ Ge, S. 1450077 \\ Ghaffar, A. 1450056 \\ Ghanbari, A. 1450088 \\ Ghosh, I. 1450030 \\ Grado-Caffaro, M. 1450045 \\ Grado-Caffaro, M. A. 1450045 \\ Gumma, S. 1450022 \\ Guo, J. X. 1450029, 1450079 \\ Guo, J. 1450032 \\ Han, H. 1450076 \\ Hang, R. 1450032 \\ Hayat, A. 1450059 \\ Hayat, S. S. 1450072 \\ He, L. 1450021, 1450024 \\ He, M. Z. 1450028 \\ He, P. $\quad 1450002,1450018$
}

He, S. Y. 1450026

He, Y. N. 1450026

Hejun, L. 1450016

Hou, L. 1450040

$\mathrm{Hu}, \mathrm{S} . \quad 1450057$

Hu, Y. 1450003

Hua, J. J. 1450069

Huang, M. 1450053

Huo, J. C. 1450079

Huo, Y.-Q. 1450053

Husain, S. A. 1450049

Huttula, M. 1450058

Hwang, P. 1450067

Islam, M. 1450059

Jayabalan, J. 1450005

Ji, D. 1450083

Ji, Z. 1450061

Jia, F. $\quad$ 1430001, 1450019

Jiang, C. H. 1450076

Jiang, D. Y. 1450015

Jiang, H. 1450074

Jiang, X. 1450018

Jiao, Y. 1450063

Jinhua, L. 1450016

Kayani, Z. N. $\quad$ 1450013, 1450081

Kezhi, L. 1450016

Khamlich, S. 1450001

Khan, D. N. 1450048

Khan, S. 1450005

Khorshidi, Z. 1450080

Kim, K.-S. 1450050

Kim, S.-S. 1450067

Kong, C. Y. 1450033

Koodali, R. T. 1450060

Kotsedi, C. K. 1450001

Kotsedi, L. 1450012

Kush, P. K. 1450005

Lai, F. 1450052

Lai, G. 1450052

Lai, S. 1450017 
Lan, J. 1450070

Le, X. 1450083

Lei, X. 1450066

Leilei, Z. 1450016

Li, C. $1450021,1450024,1450025$

Li, H. 1450061

Li, J. 1450077

Li, L. 1450065

Li, L. 1450084

Li, M. 1450006, 1450009, 1450009, 1450057, 1450077

Li, P. $\quad 1450004,1450008$

Li, W. J. 1450033

Li, W. 1450042

Li, X. H. 1450029,1450079

Li, Y. $\quad 1450014,1450070$

Liang, X. 1450042

Liao, J. 1450017

Lin, C. C. 1450069

Lin, J. G. 1450007

Lin, L. 1450052

Lin, N. $\quad 1430002,1450032,1450006$

Lin, Z. Q. 1450026

Ling, J. 1450035

Liu, B. T. $\quad 1450029,1450079$

Liu, H. 1450033

Liu, J. 1450062

Liu, L. 1450035

Liu, P. 1450014

Liu, Q. 1450031, 1450061

Liu, S. 1450035

Liu, X. 1450024, 1450025, 1450027, 1450035,1450038

Liu, Y. $\quad$ 1450027, 1450084, 1450014

Liu, Z. 1450011

Liu, Z. W. 1450069

Lu, B. 1450065

Lu, F. 1450065

Lu, Y. 1450033

Lu, Y.-P. 1450063

Luo, Z. C. 1450007

Lv, X. 1450061

Ma, D. 1450003

Ma, L. X. 1450029,1450079

Ma, P. 1450040

Ma, Y. Z. 1450028

Maaza, M. 1450001, 1450012

Madjoe, R. 1450012

Manikandan, E. 1450001

Mao, Q. 1450061

Miao, Y. 1450042

Mikhalovsky, S. V. 1450016

Mirmahalle, S. F. G. 1450073

Mishra, B. K. 1450030

Mishra, P. 1450022

Mishra, S. 1450060
Mozaffari, M. 1450047

Mubarak, A. A. K. 1450034

Mujahid, M. 1450059

Munawar, M. A. 1450048

Naseem, S. $\quad 1450013,1450081$

Nath, J. $\quad$ 1450030, 1450049

Nath, R. K. $\quad 1450030,1450049$

Naz, M. Y. 1450056

Ngom, B. D. 1450001

Niu, Y. R. 1450058

Nkosi, M. 1450012

Ouertani, R. 1450041

Ouyang, H. 1450025

Pal, A. 1450030

Pal, S. 1450005

Pan, W. 1450018

Pankratov, V. 1450058

Peng, C. 1450019

Peng, R. 1450060

Peng, W. 1450007

Phan, D.-T. 1450086

Podder, J. 1450044

Przybylowicz, W. 1450012

Qiang, S. 1450016

Qin, L. 1450006

Qiu, J. 1450017

Qu, Y. 1450052

Rahman, S. S. 1450072

Ran, R. 1450087

Rasheed, H. 1450059

Rashidi, M. M. 1450036

Rehman, Z. 1450072

Ren, G. 1450014

Ren, J. $\quad 1450002,1450018$

Ren, T. 1450038

Riaz, S. $\quad 1450013,1450081$

Sagar, A. K. 1450005

Sagir, M. 1450056

Sahari, A. 1450082

Sahu, D. 1450022

Salam, S. 1450059

Samuel, E. 1450046

Sandt, C. 1450012

Sayyad, M. H. 1450048

Sechogela, T. 1450012

Segu, D. Z. 1450067

Shah, Z. A. 1450072

Shakir, I. 1450056

Shanak, H. 1450043

Sharma, T. K. 1450005
Shen, B. $\quad$ 1450037, 1450051, 1450066, 1450068, 1450078

Sheng, C. 1450016

Shi, J. 1450010

Shirmane, L. 1450058

Shukrullah, S. 1450056

Sima, W. 1450010

Sohail, A. 1450036

Song, S. $\quad 1430001,1430003,1450019$

Song, X. 1450025

Song, Y. 1450038

Song, Z. 1450017

Sui, H. 1450064

Sun, F. $\quad 1450037,1450051,1450066$, 1450068, 1450078

Sun, Y. 1450077

Sun, Z. 1450070

Tahir, M. 1450048

Tang, B. 1430002, 1450006, 1450032

Tang, D. M. 1450007

Tang, X. 1450065

Tao, X. W. 1450085

Tian, D. X. 1450033

Uddin, A. S. M. I. 1450086

Ullah, S. 1450056

Verma, A. 1450022

Wahab, F. 1450048

Wajid, H. A. 1450036

Wang, F. 1450058

Wang, G. 1450064

Wang, J. 1450003, 1450009

Wang, L. 1450025, 1450035, 1450037

Wang, M. 1450031

Wang, S. J. 1450079

Wang, T. 1450042

Wang, X. 1450066, 1450070, 1450077

Wang, Y. $\quad 1450035,1450053$

Wang, Y. L. 1450029

Wang, Y. M. 1450007, 1450085

Wang, Z. 1450025, 1450031, 1450035

Wang, Z. Z. 1450085

Wei, G. $\quad$ 1450057, 1450009

Wei, L. 1450016

Wei, S. 1450035

Williams, R. V. 1450020

Wu, C.-M. 1450060

Wu, F. 1450033

Wu, H. 1450017

Wu, J. 1450018 
Wu, W. 1450069

$\mathrm{Wu}, \mathrm{X} . \quad 1450061$

Wu, X. Q. 1450007

Wu, Y. 1450052

Xi, J. 1450061

Xiang, F. 1450025

Xiang, G. S. 1450074

Xiao, M. 1450042

Xiao, P. 1450087

Xie, L. 1450055

Xie, Z. 1450028

$\mathrm{Xu}, \mathrm{S} . \quad 1450057$

$\mathrm{Xu}, \mathrm{X} . \quad 1450035$

$\mathrm{Xu}, \mathrm{Y} . \quad 1450040$

$\mathrm{Xu}, \mathrm{Y} . \mathrm{F} . \quad 1450074$

Xue, D. J. 1450015

Yakovkin, I. N. 1450039

Yang, B. 1430003

Yang, C. 1450064

Yang, H.-Y. 1450063

Yang, L. 1450079

Yang, Q. 1450010, 1450014
Yang, Y. 1450017, 1450038, 1450042, 1450057

Yang, Z. 1450017, 1450042

Yaseen, M. 1450048

Yi, J. 1450071

Yin, Z. 1450017

$\mathrm{Yu}$, H. J. 1450026

$\mathrm{Yu}, \mathrm{X} . \quad 1450070$

Zahedi, E. 1450047

Zarroug, A. 1450041

Zeng, Y. 1450069

Zhan, K. 1450076

Zhang, D. C. 1450007

Zhang, H. 1430002

Zhang, H. G. 1450055

Zhang, H.-B. 1450062

Zhang, J. Z. 1450055

Zhang, L. 1450029

Zhang, M.-X. 1450053

Zhang, S. F. 1450033

Zhang, X. 1450003, 1450006

Zhang, X. B. 1450085

Zhang, X. Y. 1450079
Zhang, Y. 1450014

Zhang, Y.-L. 1450063

Zhang, Z. 1450037

Zhao, H. D. 1450029

Zhao, M. 1450070

Zhao, Q. X. 1450029

Zhao, S. 1450031

Zhao, X. 1450009, 1450057

Zhao, Y. J. 1450029

Zheng, X. 1450023

Zheng, X. B. 1450069

Zheng, X. J. 1450076

Zhong, Q. 1450083

Zhou, D. 1450017

Zhou, H. W. 1450015

Zhou, Q. 1450083

Zhou, Y. M. 1450028

Zhu, H. J. 1450029

Zhu, L. 1450035

Zhu, Q. 1450002

Zouaoui, A. 1450082

Zou, J. $1430002,1450032,1450006$ 